\title{
Lidocaine Inhibits Myoblast Cell Migration and Myogenic Differentiation Through Activation of the Notch Pathway
}

This article was published in the following Dove Press journal:

Drug Design, Development and Therapy

\author{
Xiangtian Ling $\mathbb{D}^{\prime}$ \\ Xinqi Ma' \\ Xielan Kuang $\mathbb{D}^{\prime}$ \\ Yuxiu Zou' \\ Han Zhang (D) \\ Han Tang' \\ Han Du ${ }^{1}$ \\ Binbin Zhu' \\ Hao Huang' \\ Qing Xia' \\ Minghao Chen (ID) \\ Danyi Mao' \\ Dongli Chen' \\ Huangxuan Shen (1D ${ }^{1,2}$ \\ Jianhua Yan'
}

'State Key Laboratory of Ophthalmology, Zhongshan Ophthalmic Center, Sun YatSen University, Guangzhou, 510060, People's Republic of China; ${ }^{2}$ Biobank of Eye, State Key Laboratory of Ophthalmology, Zhongshan Ophthalmic Center, Sun Yat-Sen University,

Guangzhou, 510060, People's Republic of China

Correspondence: Huangxuan Shen; Jianhua Yan

Zhongshan Ophthalmic Center, Sun Yat-Sen University, 54 Xianlie Road, Guangzhou,

510060 , People's Republic of China

Tel +8620-8733-526I; +8620-8733-0484

Fax +8620-8733-327I

Email shenhx@mail.sysu.edu.cn;

yanjh20II@I26.com
Purpose: To assess the cellular and molecular effects of lidocaine on muscles/myoblasts.

Methods: Cultured myogenic precursor $(\mathrm{C} 2 \mathrm{C} 12)$ cells were treated with varying concentrations of lidocaine.

Results: Cell viability of $\mathrm{C} 2 \mathrm{C} 12$ cells was inhibited by lidocaine in a concentrationdependent manner, with concentrations $\geq 0.08 \%$, producing a dramatic reduction in cell viability. These $\geq 0.08 \%$ concentrations of lidocaine arrested cell cycles of $\mathrm{C} 2 \mathrm{C} 12$ cells in the $\mathrm{G} 0 / \mathrm{G} 1$ phase. Moreover, lidocaine inhibited cell migration and myogenic processes in C2C12 cells at low concentrations. Results from QRT-PCR assays revealed that following treatment with lidocaine, Notch1, Notch2, Hes1, Cs1 and D114 all showed higher levels of expression, while no changes were observed in Mmal1, Hey1, Dll1 and Jag1.

Conclusion: This work provides the first description of the effects of lidocaine upon the regeneration of muscles and maintenance of satellite cells at the cellular and molecular levels. In specific, we found that the Dll4-Notch-Csl-Hes1 axis was up-regulated suggesting that the Notch signaling pathway was involved in producing these effects of lidocaine. These findings provide a new and important foundation for future investigations into the effects of drug therapies in muscle diseases.

Keywords: local anesthetics, $\mathrm{C} 2 \mathrm{C} 12$ cells, myogenic differentiation, strabismus, Notch signaling pathway

\section{Introduction}

Recently, local anesthetics, which are typically utilized as analgesic agents through their capacity to block sodium channels, have found other applications such as producing antiarrhythmic and antineoplastic effects, ${ }^{1,2}$ and in the treatment of strabismus. With strabismus, a disruption in the strength of an extraocular muscle (EOM) is a key factor in misaligning eye motion. ${ }^{3}$ Treatment with local anesthetics can modulate this muscle force ${ }^{4}$ to induce changes in muscle biomechanics, ${ }^{5}$ thus rectifying the strabismus.

Results from several studies have indicated that local anesthetics, including bupivacaine, ropivacaine and lidocaine, exert myotoxicities which are similar in nature, ${ }^{6,7}$ usually resulting in lytic degeneration of striated muscle sarcoplasmic reticulum, myocyte edema and necrosis. ${ }^{8}$ It has been reported that the mechanisms involved with such muscle injuries are, in part, attributable to aberrations in cytoplasmic calcium homeostasis by sarcoplasmic reticulum $\mathrm{Ca}^{2+} /$ ATPase processes, ${ }^{9}$ effects which may be mediated by mitochondria. ${ }^{10}$ 
However, the exact mechanisms regarding local anesthetic affects upon muscles and the modulation of muscular forces are still not fully understood. One hypothesis that has been proposed to explain the effects of local anesthetics in strabismus treatment is that there is a selective damage to muscle fibers of the EOMs while preserving cellular support structures, nerves and satellite cells. ${ }^{11}$ Subsequently, these satellite cells activate myoblasts to initiate a rapid process of regeneration. ${ }^{12}$ While it is not entirely clear as to the means through which local anesthetics affect satellite cells or myoblasts, there is evidence from previous studies that these local anesthetics can be cytotoxic to myoblasts through their ability to induce anti-proliferation and cell death. ${ }^{13,14}$ In this study, we used the $\mathrm{C} 2 \mathrm{C} 12$ cell line to study the effects of one commonly used local anesthetic, lidocaine, upon muscle satellite cells as an approach to understand some of the mechanisms of muscle regeneration after treatment with local anesthetics.

\section{Materials and Methods}

\section{Reagents and Plasmids}

Lidocaine was purchased from Aladdin (Shanghai, China).

The following antibodies were used for our Western blot assays, myosin heavy chain 3 (1:1000, Abcam, ab124205), Myogenin (1:1000, Absin, ab137750), Pax7 (1:250, Abcam, ab199010), Notch1 (1:1000, Cell Signaling Technology, \#3439), Notch2 (1:1000, Cell Signaling Technology, \# 5732S), Hes1 (1:200, Santa Cruz Biotechnology, sc-166378) and $\beta$-actin (1:2000, Bioworld Technology, Inc., AP0060).

Plasmids for the reporter genes of Myog-luc, ${ }^{15} \mathrm{MCK}-$ luc, ${ }^{16} 3 \times$ MEF2-luc, Csl-luc ${ }^{17}$ and pRL-TK were used for the luciferase assay.

\section{Cell Culture and Differentiation Induction}

$\mathrm{C} 2 \mathrm{C} 12$ cells were kindly provided by Cell Bank/Stem Cell Bank, Chinese Academy of Sciences. C2C12 cells were cultured in Dulbecco's modified Eagle's medium (DMEM) medium. Details regarding culture and differentiation conditions have been described previously. ${ }^{18}$ Briefly, C2C12 cells were maintained in DMEM high glucose (Gibco; Thermo Fisher Scientific, Inc., Waltham, MA, USA) supplemented with $20 \%$ fetal bovine serum (HyClone; GE Healthcare Life Sciences, Logan, UT, USA), 100 U/mL of penicillin. The differentiation of $\mathrm{C} 2 \mathrm{C} 12$ cells was induced by DMEM high glucose with $2 \%$ horse serum (Gibco).

\section{Wound Healing, RT-PCR and Luciferase Assays}

The $\mathrm{C} 2 \mathrm{C} 12$ cells were cultured in 12-well plates, which were used for the wound healing assay when densities achieved $80 \%$.

RT-PCR was utilized to identify relative expression levels of the following genes: Myod1, Myog, Notch1, Notch2, Hes1, Csl, Dl14, Pax3, Pax7, Myf5, Six1 and Six4. The sequences of forward and reverse chains were obtained from PrimerBank (https://pga.mgh.harvard.edu/ primerbank/). The primers were synthesized by SangonBiotech (Guangzhou, China).

The luciferase assay was performed as described previously. ${ }^{19,20}$ Briefly, Lipofectamine $^{\text {TM }} \quad 3000$ (Invitrogen, Carlsbad, CA, USA) was used in transfection, following the manufacturer's instructions. $\mathrm{C} 2 \mathrm{C} 12$ cells were harvested after $48 \mathrm{~h}$ transfection, and luciferase activity was measured with the Dual-Glo Luciferase Assay System (Promega, Madison, WI, USA)

\section{Apoptosis Measurements, Cell Cycle Assay and Cell Viability Determinations}

The Cell Counting Kit-8 (CCK8, Dojindo, Japan) was utilized to determine cell viability. Apoptosis of $\mathrm{C} 2 \mathrm{C} 12$ cells, as assessed in the presence or absence of lidocaine, was performed with the use of the Multi-caspase Kit (Millipore, Cat: MCH100109, USA). PI/RNase Staining Buffer (BD Biosciences Pharmingen, Cat:550825, USA) was utilized in the cell cycle assay.

\section{Human Extraocular Primary Myoblasts}

The isolation and culture of primary myoblasts as obtained from strabismus surgery samples were performed as described previously. ${ }^{21}$ The myoblasts were maintained in HAM'S F10 - MEDIUM (Gibco) with $20 \%$ heat inactive fetal bovine serum.

\section{Statistical Analysis}

Each experiment was repeated at least three times. The IBM SPSS Statistics 20 software program was used for conducting the statistical analysis. The results are displayed as means \pm standard deviations. Statistically significant differences among the groups were assessed using a one-way ANOVA followed by the LSD post hoc test for 
pairwise comparisons. Probability levels obtained with

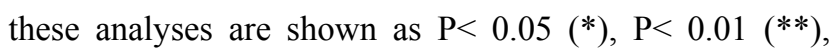
$\mathrm{P}<0.001(* * *)$ and $\mathrm{P}>0.05(\#)$.

\section{Results}

\section{High Concentrations of Lidocaine Induce} Apoptosis and Inhibit Cell Proliferation in $\mathrm{C} 2 \mathrm{Cl} 2$ Cells

We first determined cell viability in response to varying concentrations of lidocaine $(0-25 \%, 1 \%=34.6 \mathrm{mmol} / \mathrm{L})$ for 8 or $24 \mathrm{~h}$. Results of the CCK 8 assay revealed that lidocaine produced a concentration-dependent decrease in cell viability, with high concentrations significantly inhibiting myoblast viability (Figure 1A). The Multi-caspase Kit was then used to determine apoptosis in these $\mathrm{C} 2 \mathrm{C} 12$ cells in response to an $8 \mathrm{~h}$ exposure of varying lidocaine concentrations. Similar to that observed for cell viability, there was a concentration-dependent increase in cell apoptosis with high concentrations inducing significant levels of apoptosis in these $\mathrm{C} 2 \mathrm{C} 12$ cells (Figure $1 \mathrm{~B}$ and $\mathrm{C}$ ).

\section{Lidocaine Induces Cell Cycle Arrest of C2CI 2 Cells}

We next investigated which periods of the cell cycle were affected by lidocaine. After incubating C2C12 cells for 24 $\mathrm{h}$ with different concentrations of lidocaine $(0,0.02,0.04$,

\section{CCK8 8h}

A

B

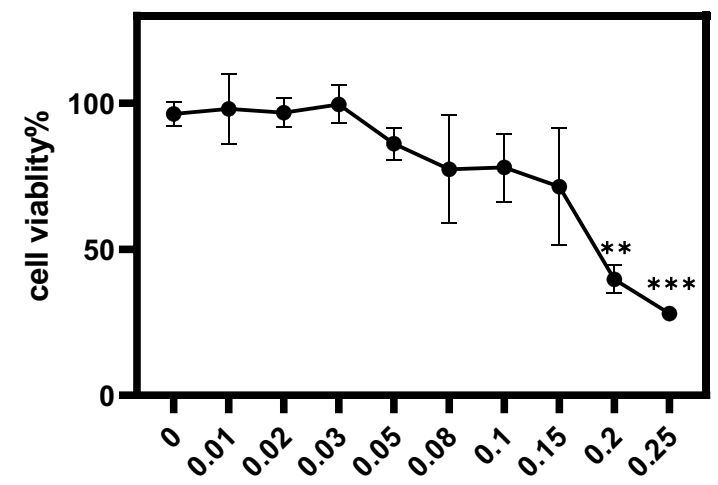

Concentration of LID(\%)

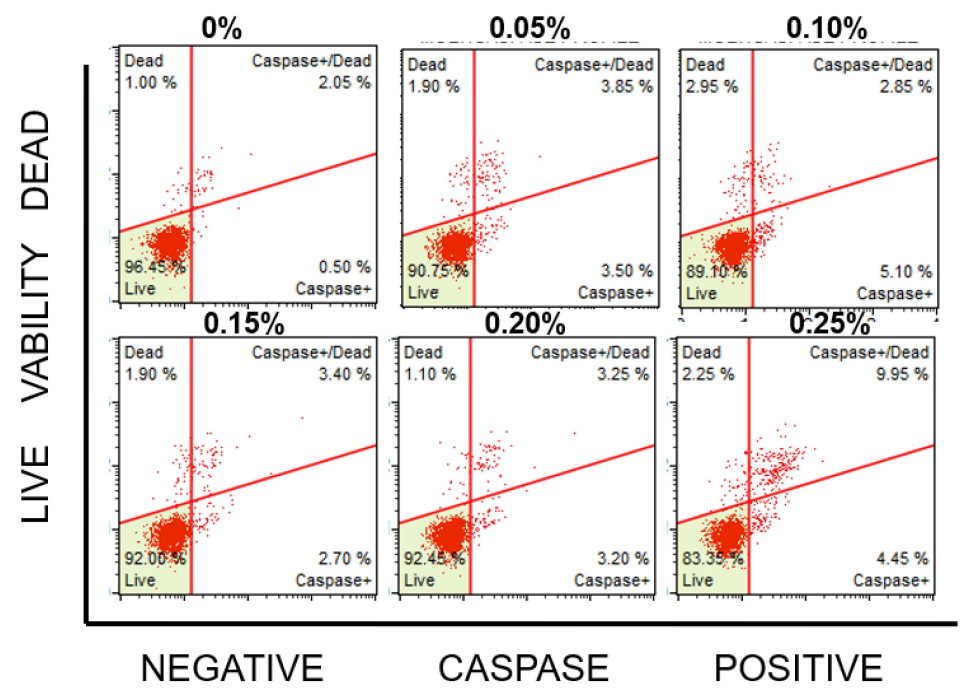

CCK8 24h

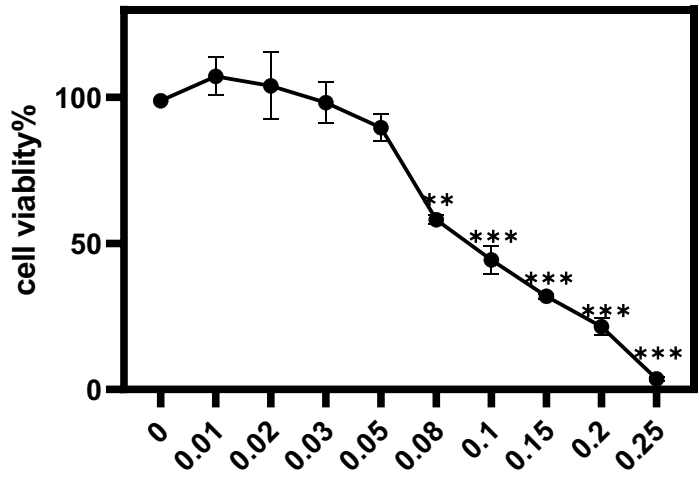

C

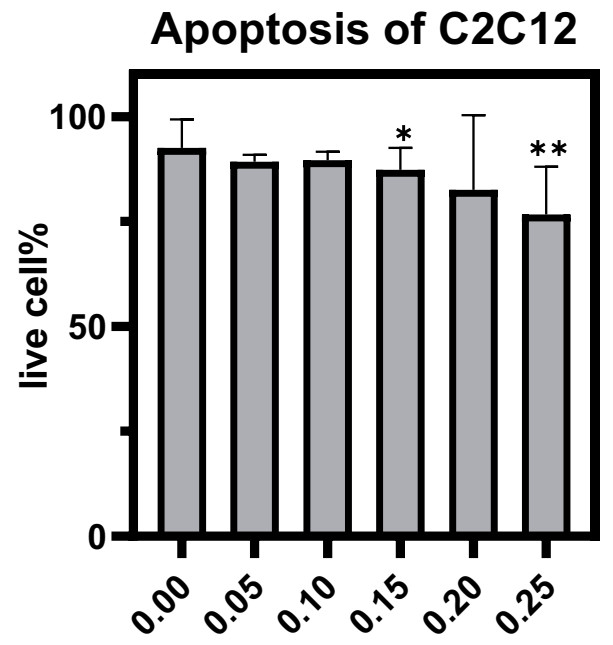

Concentration of LID(\%)

Figure I Lidocaine (LID) induces apoptosis in $\mathrm{C} 2 \mathrm{Cl} 2$ cells. (A) $\mathrm{C} 2 \mathrm{Cl} 2$ cells were treated with different concentrations of lidocaine ranging from $0 \%$ to $0.25 \%$ for 8 or 24 $\mathrm{h}$ and cell viability was determined with use of the CCK8 assay. Lidocaine inhibited cell viability in a concentration-dependent manner. (B) C2CI 2 cells were treated with different concentrations of lidocaine ranging from $0 \%$ to $0.25 \%$ for $8 \mathrm{~h}$ and the percent of live and apoptotic cells were determined with use of flow cytometry. (C) Bar graph representation for quantification of results from $(\mathbf{B})$ showing that cell apoptosis increased in a concentration-dependent manner to lidocaine. Each experiment was repeated three times. For lidocaine: $1 \%=34.6 \mathrm{mmol} / \mathrm{L}(* P<0.05, * * P<0.01, * * * P<0.001, \mathrm{n}=3$, bars represent $\mathrm{SD})$. 
$0.06,0.08,0.10 \%$, these cells were stained using $\mathrm{PI} /$ RNase Staining Buffer. Flow cytometry was then used to measure the percent of $\mathrm{C} 2 \mathrm{C} 12$ cells within the different cycles. With lidocaine concentrations of $\geq 0.08 \%$, we found, that the cell cycle was arrested at the G0/G1 phase (Figure 2A and B). Lower concentrations of lidocaine failed to significantly alter these cell cycles.

\section{Low Concentrations of Lidocaine Inhibit Migration of $\mathrm{C} 2 \mathrm{Cl} 2$ Cells and Human Extraocular Primary Myoblasts}

As migration of myoblasts represents a critical component for muscle regeneration, we were interested in examining the effects of lidocaine in this process. C2C12 cells were planted in 12-well plates and a wound was induced at the density of $80 \%$. For this experiment, lidocaine concentrations from $0 \%$ to $0.06 \%$ were used to preclude the possibility for $\mathrm{C} 2 \mathrm{C} 12$ cell apoptosis and proliferation and the FBS was reduced to $3 \%$. Photos were taken at 0,24 and $48 \mathrm{~h}$ post-lidocaine treatment (Figure 3A), and the area occupied by the cells was calculated within each photo (Figure $3 \mathrm{~B}$ ). We found that $\mathrm{C} 2 \mathrm{C} 12$ cell migration was inhibited by lidocaine at these low concentrations. Similar results were obtained when testing these effects of lidocaine in primary culture myoblasts of human EOMs (Figure $3 \mathrm{C}$ and $\mathrm{D}$ ). The myogenic differentiation test was used to verify that the primary culture cells were myoblasts (Figure S1).

\section{Low Concentrations of Lidocaine Inhibit C2CI2 Cell Differentiation}

As an approach to determine whether differentiation of myoblasts would be affected by this local anesthetic, a differential experiment was performed. For this experiment, we changed the growth medium to differentiation medium $(3 \%$ horse serum in DMEM), then treated these cells with $0 \%, 0.01 \%$ or $0.02 \%$ lidocaine and assessed the morphology of these cells. Photos were then taken on the fourth day of differentiation. In the control group, cells gradually merged into mature myotubes and, morphologically, achieved the shape of mature myotubes with large and long bars, instead of spindleshaped mononuclear cells. In the treatment group, many mononuclear cells and immature myotubes remained present. These results suggest that even at quite low concentrations, incapable of affecting apoptosis and proliferation in these $\mathrm{C} 2 \mathrm{C} 12$ cells, lidocaine inhibited the differentiation of these cells (Figure 4A). To investigate the promoter activity of differentiation marker genes potentially associated with this effect, including Mef, Myogenin, Mef2c and MCK, we performed a luciferase assay (Figure 4B). We found that the priming of genes, Mef, Mef2c and Myogenin, were decreased by this lidocaine treatment.

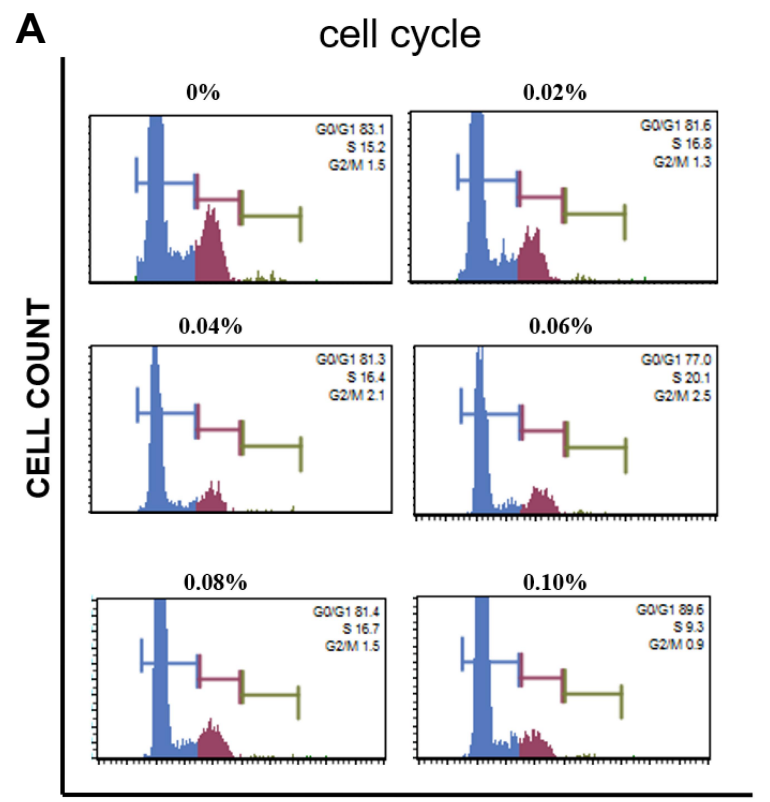

B

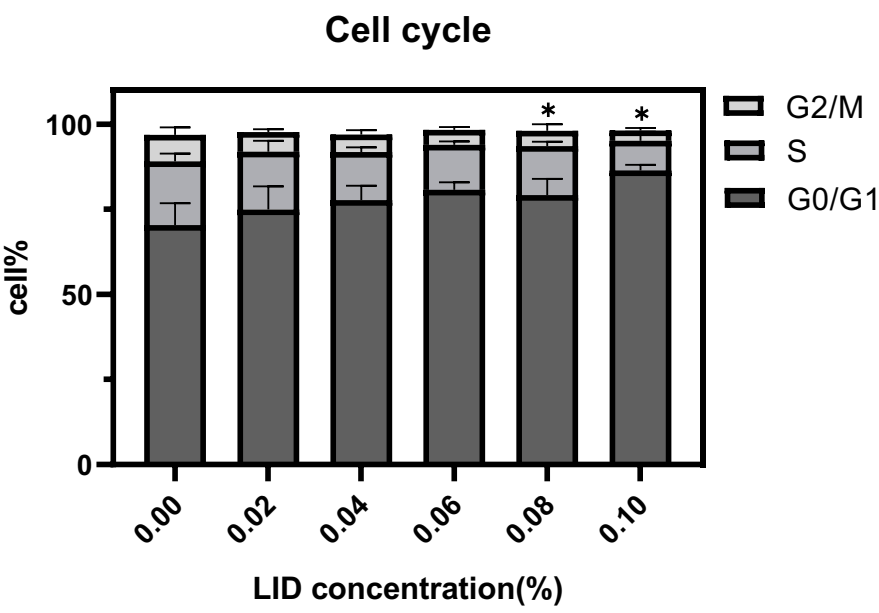

DNA CONTENT INDEX

Figure 2 Lidocaine induces cell cycle arrest. (A) Flow cytometric analysis of the cell cycle. (B) Bar graph representation for quantification of results from (A). At concentrations $\geq 0.08 \%$, lidocaine arrested the cell cycle at the $G 0 / G I$ phase $\left({ }^{*} P<0.05, n=3\right.$, bars represent $\left.S D\right)$. 

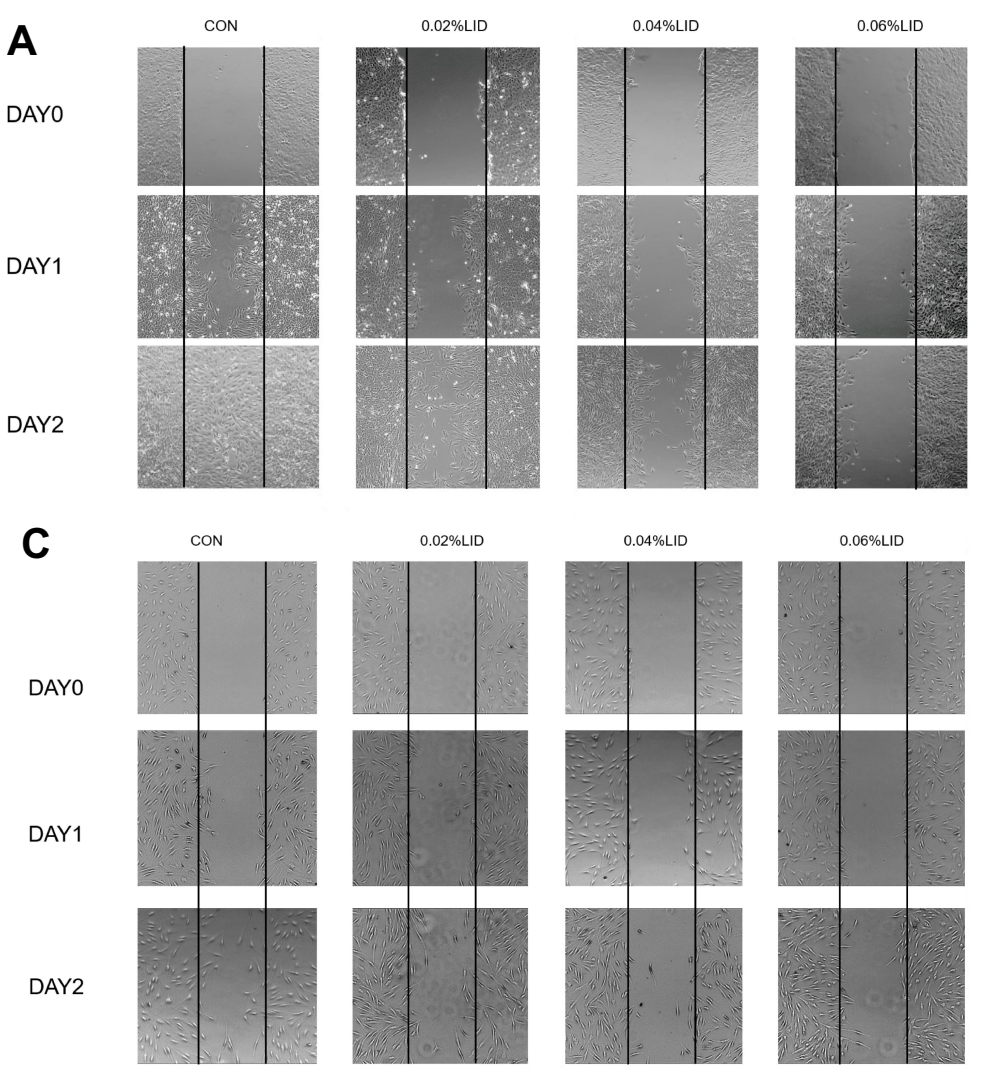

B Would healing of $\mathrm{C2C} 12$ myoblasts

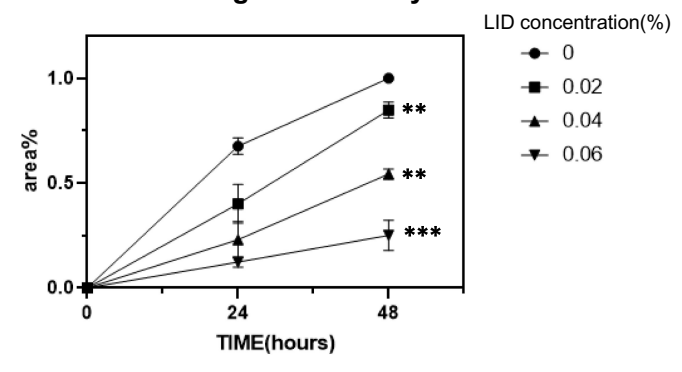

D

Would healing of primary culture EOM myoblasts

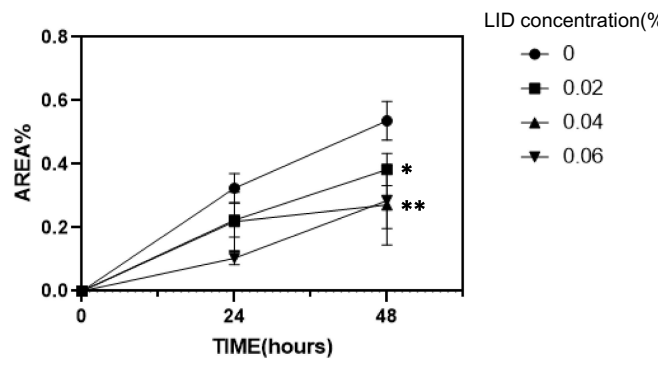

Figure 3 Low concentrations of lidocaine inhibit migration of $\mathrm{C} 2 \mathrm{Cl} 2$ cell and satellite cell (SC). (A) Planted $\mathrm{C} 2 \mathrm{Cl} 2$ cells in 24 -well plate and created wound at the density of $80 \%$. The cells were then treated with lidocaine at concentrations ranging from 0 to $0.06 \%$ and photos were taken at 0,24 and $48 \mathrm{~h}$ post-lidocaine treatment. (B) Area occupied by $\mathrm{C} 2 \mathrm{Cl} 2$ cells within the two black lines was calculated from images. (C) Extraocular muscle satellite cells in 24 -well plate and created wound at the density of $80 \%$. The cells were treated with lidocaine at concentrations ranging from 0 to $0.06 \%$ and photos were taken at 0,24 and $48 \mathrm{~h}$ post-lidocaine treatment. (D) Area occupied by extraocular muscle satellite cells within the two black lines was calculated from images. $(* \mathrm{P}<0.05, * * \mathrm{P}<0.01, * * * \mathrm{P}<0.00 \mathrm{I}, \mathrm{n}=3$, bars represent $\mathrm{SD})$.

Subsequently, RT-PCR was used to measure mRNA levels of Myod1 and Myog, two critical differential control genes. Both showed significant decreases in response to lidocaine, even at a concentration of $0.01 \%$. At the protein level, results of Western blot of MYH3, a marker of muscle differentiation, confirmed that the myogenic differentiation was inhibited.

\section{Low Concentrations of Lidocaine May Maintain Quiescence of Myoblasts via the Notch Signaling Pathway}

To further investigate the mechanisms involved through which lidocaine inhibits differentiation of myoblast, the effects of lidocaine on immunofluorescence of $\mathrm{C} 2 \mathrm{C} 12$ cells were assessed. After 2 days of treatment with differentiation medium (with or without lidocaine), myosin antibodies were used to mark differentiated cells. In response to $0.02 \%$ lidocaine, many myoblasts remained undifferentiated (Figure 5A). Luciferase assay results revealed and found that the priming of Csl, the Notch effector, increased when treated with a low concentration of lidocaine (Figure 5B). RT-PCR was then used to investigate the relative expression levels of Notch signaling pathway genes. We found that Notch1, Notch2, Hes1, Cs1 and Dll4 were all expressed in high levels (Figure 5C), while levels of Mmal1, Hey1, Dll1

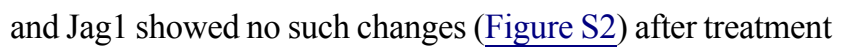
with low concentrations of lidocaine. Similar findings were obtained from results of Western blot assays (Figure 5D, $\mathrm{E}$ and $\underline{\mathrm{S} 4 \mathrm{~B}}$ ). These results suggest that lidocaine may affect myoblasts via the Notch signaling pathway. Moreover, to figure out which molecule lidocaine acts on, we silenced Dl14, Notch1 and Notch2, respectively, with siRNA (purchased from RiboBio, Guangzhou, China) to observe the effect of lidocaine on Notch pathway. When we silenced Dll4 (Figure S3A), the Notch ligand, the effect that low concentration of lidocaine activates Notch pathway disappeared. On the other hand, when Notch1 or Notch2, two different types of Notch receptors, was silenced, the expression level of Dll4 could be up-regulated by lidocaine (Figure $\underline{\mathrm{S} 3 \mathrm{~B}}$ and $\underline{\mathrm{C}})$. These results showed that D114 is the most likely 
A

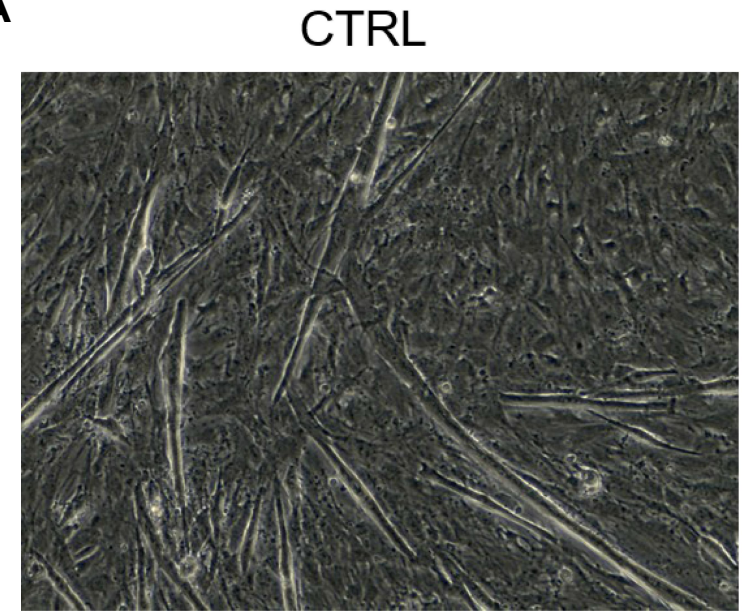

\section{$0.02 \%$ LID}

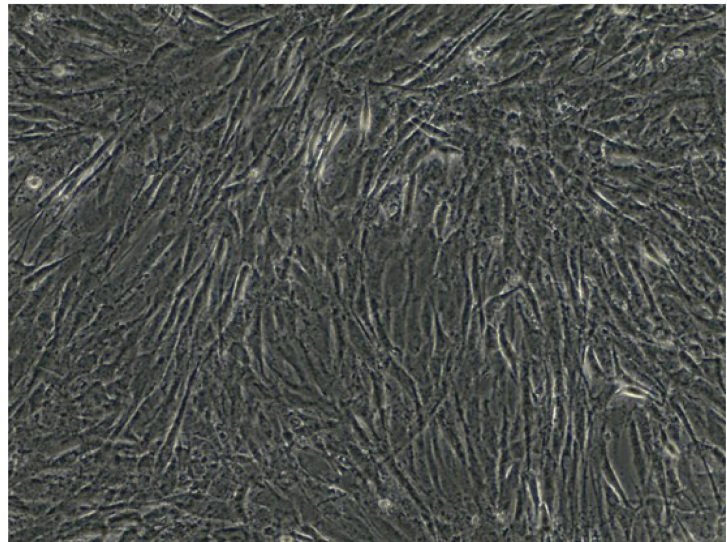

B

Myog-luc

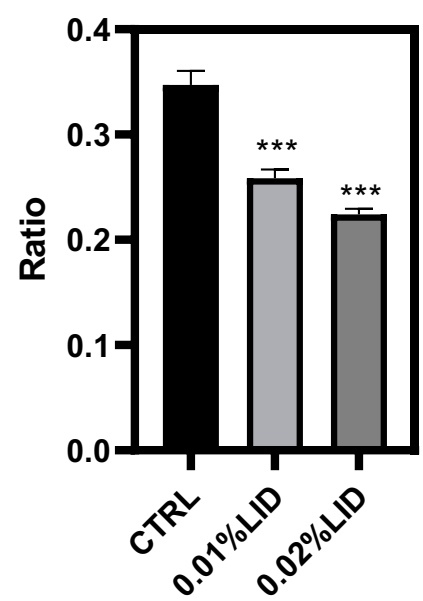

C

Myod1

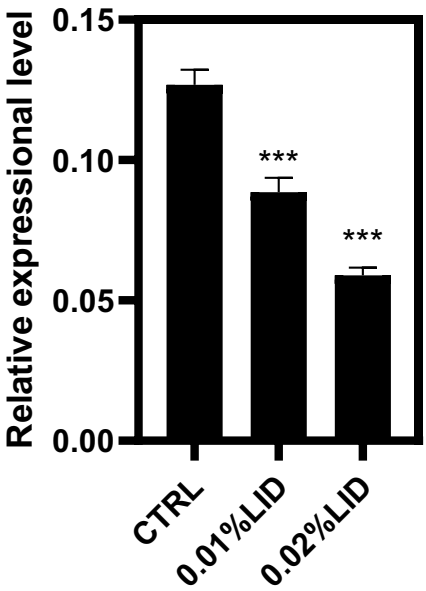

3*MEF-Iuc

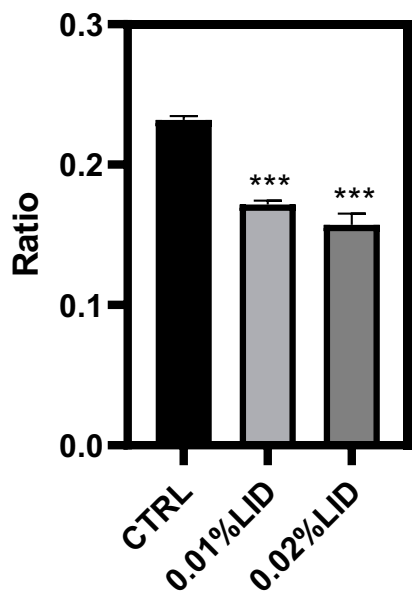

Myog

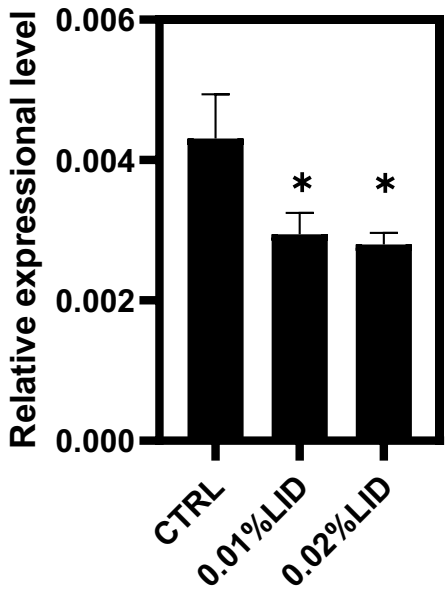

3*MEF2c-luc

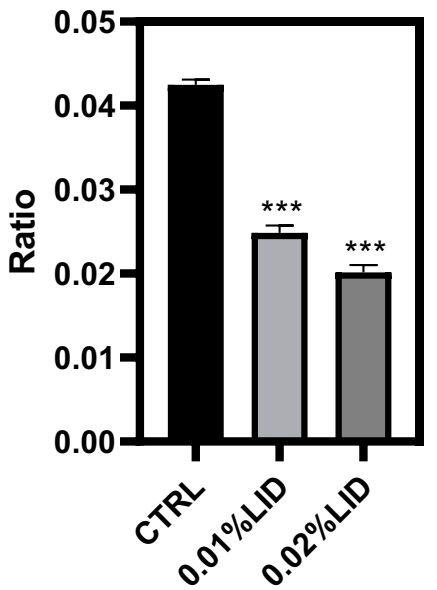

D

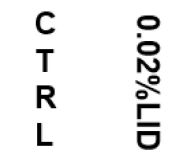

Myh3

$\beta$-actin

Myogenin

$\beta$-actin
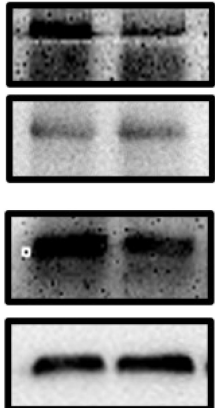

Figure 4 Low concentrations of lidocaine inhibit $\mathrm{C} 2 \mathrm{Cl} 2$ cell differentiation. (A) $\mathrm{C} 2 \mathrm{Cl} 2$ myoblasts as induced in differential medium and treated with or without $0.02 \%$ lidocaine. Photos were taken on the fourth day. (B) 3xMef-luc, 3xMef2c-luc and Myog-luc were co-transfected with pRL-TK. At 24 h after transfection, lidocaine at concentrations of $0,0.01,0.02 \%$ were added and luciferase activity was then determined after $48 \mathrm{~h}$ of incubation. (C) qRT-PCR was used to measure transcript levels of the muscle-specific genes, Myog and MyodI. RNA samples were collected at two days after differentiation. (D) Two days after differentiation, protein levels of Myh3 were determined using Western blot. ( $* \mathrm{P}<0.05$, $* * * \mathrm{P}<0.001, \mathrm{n}=3$, bars represent $\mathrm{SD})$. 
A
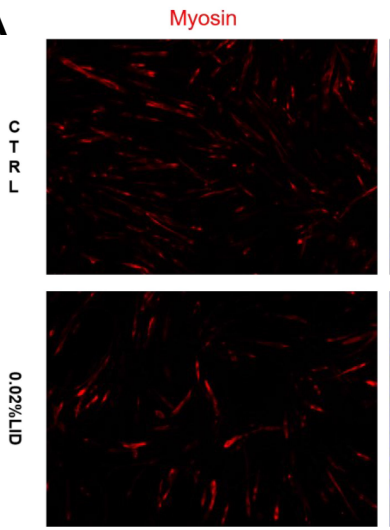

Hoechst
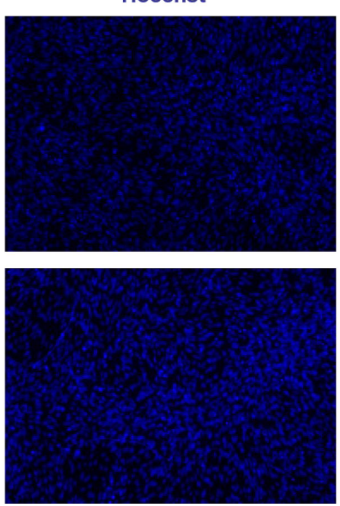
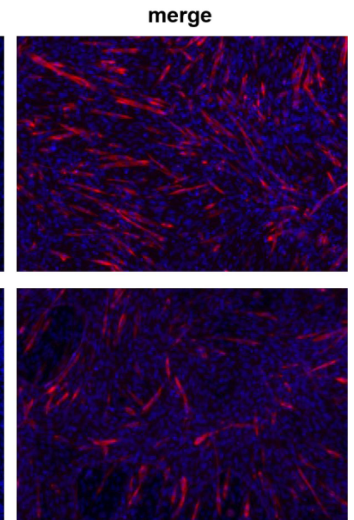

B

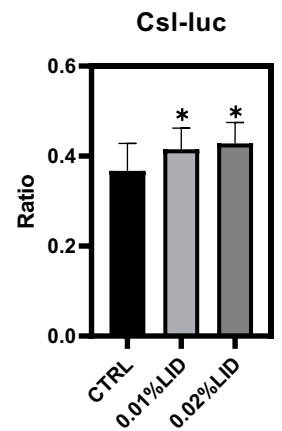

D

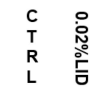

Hes1

Notch1

Notch2

$\beta$-actin

Pax7

$\beta$-actin

\section{C}

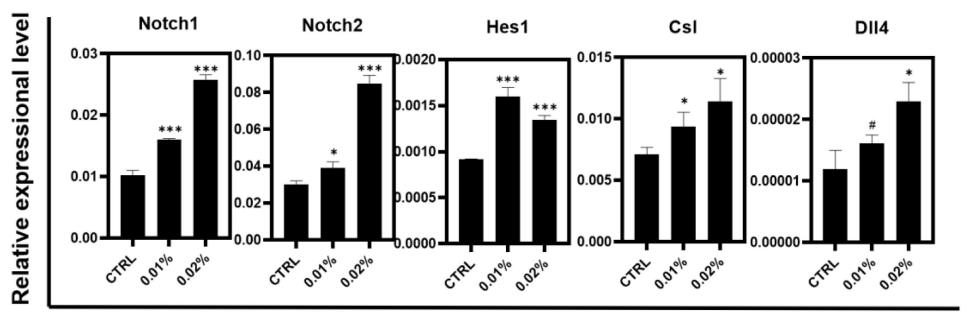

$F$

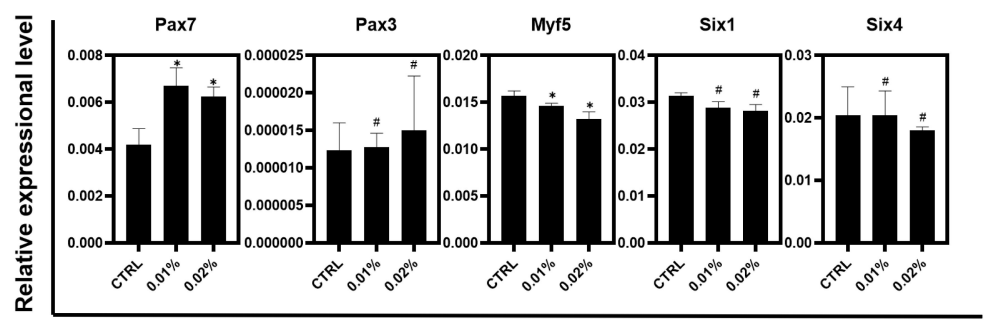

E

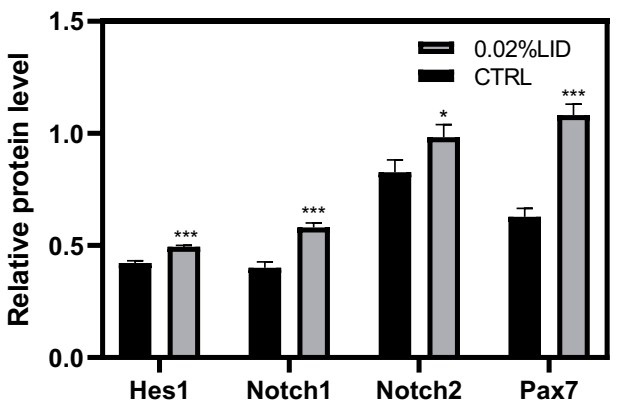

Figure 5 Low concentration of lidocaine may inhibit activation of myoblasts via Notch signaling pathway. (A) Immunofluorescence was used to mark the differentiated cells. Cells were fixed two days after differentiation. (B) Csl-luc were co-transfected with pEF-RL, respectively. $24 \mathrm{~h}$ after transfection, $0,0.0 \mathrm{I}, 0.02 \%$ concentration of lidocaine was added. Luciferase activity was measured after $48 \mathrm{~h}$ incubation. (C) qRT-PCR was used to measure the transcript levels of Notch signaling pathway genes Notchl, Notch2, Hes I, Csl, DIl4. RNA samples were collected two days after differentiation. (D) Protein level of Hes I, Notch I, Notch2, Pax7 was analyzed by Western blot. Protein samples were collected two days after differentiation. (E) Bar graph is for quantification of figure 5D. (F) qRT-PCR was used to measure the transcript levels of muscle lineage genes: Pax3, Pax7, Myf5, Sixl, Six4. RNA samples were collected two days after differentiation. (\#P>0.05, $* P<0.05, * * * P<0.001, n=3$, bars represent SD).

molecule that lidocaine acts on. What is more, up-regulating of Dll4-Notch1-Cs1-Hes1 axis was still observed with the treatment of si-Notch2, which means that Notch2 is not necessary when lidocaine activating Notch pathway (Figure $\underline{\mathrm{S} 3 \mathrm{~B}}$. If Notch1 is silenced, lidocaine only up-regulates D114 but does not up-regulate Hes1, the Notch target (Figure S3C). This result reveals that Notch1 is an indispensable molecule in this pathway when myoblasts are treated with lidocaine.

To corroborate these results on the differentiation stages of the myoblasts, RT-PCR was used to measure some of the critical transcription factors regulating progression through the myogenic lineage. ${ }^{22}$ We found that Pax 3 and Pax 7 showed relatively increased expression levels in response to lidocaine (Figure 5F), which suggests that more myoblasts remained either undifferentiated or in the early stages of differentiation.

\section{Discussion}

The myotoxicity of local anesthetics (LAs), first described in 1959 by Brun, ${ }^{9}$ is a complication of peripheral nerve blocks. Subsequent work as performed in animal models was directed toward studying the means by which LAs damage tissue. ${ }^{23}$ In spite of this potential for damage, treatment of EOMs with LAs has proved effective in correcting strabismus. After injection with LAs, muscles initially show degeneration and inflammation followed by regeneration with increased diameters of muscle fibers. ${ }^{24}$ Satellite cells play a key role in this process. In this study, we investigated how one specific LA, lidocaine, affects satellite cells under laboratory conditions. First, we established that this LA would induce apoptosis of $\mathrm{C} 2 \mathrm{C} 12$ cells, in a dose- and time-dependent manner, as had been 
reported by others. ${ }^{13,25}$ At a concentration of $>0.08 \%$, we observed dramatic reductions in cell viability and the halfmaximal inhibitory concentrations $\left(\mathrm{IC}_{50}\right)$ were calculated as $0.099 \% \pm 0.004 \%$ (Figure S4A). These results were similar to that of Maurice et $\mathrm{al}^{13}$ who reported an $\mathrm{IC}_{50}$ of lidocaine to be $0.097 \% \pm 0.015 \%$. The novel findings of our current study were the first demonstration that lidocaine could inhibit the migration of $\mathrm{C} 2 \mathrm{C} 12$ cells at concentrations as low as $0.02 \%$, concentrations that would not affect cell viability or the cell cycle. This result suggests that even with the use of a dose considered safe, lidocaine may inhibit the migration of myoblasts. Normally, skeletal muscle repair requires migration of myoblasts, both to the injury site and then within the wound. ${ }^{26}$ The inhibition of migration by lidocaine, as observed here, indicates that myoblasts would be incapable of the transport necessary to promote muscle repair. Such an effect may explain the basis for the incomplete repair accompanied by connective tissue that sometimes occurs after LA injection.

Myogenic differentiation is critical for muscle repair. Following acute injury, functional muscle fibers are usually destroyed. Then, satellite cells, stimulated by pathogenic factors or inflammation, are transformed into activated myoblasts which are transported to the injury site to initiate the repair process. These myoblasts proliferate, differentiate, fuse into mature multinucleated myotubes and finally form functional fibers. ${ }^{22}$ In this study, we demonstrated that lidocaine could inhibit myogenic differentiation at concentrations that would not affect cell viability or proliferation. In this way, muscle repair would be perturbed, which may, in part, explain the myotoxicity of LAs. The diplopia or strabismus complications that can sometimes occur with anterior and posterior segment eye surgery ${ }^{27}$ may result from the capacity of LAs to inhibit the migration and differentiation of myoblasts.

According to Maurice's study, activation of Akt and ERK pathways is diminished obviously at a high concentration of local anesthetics. ${ }^{13}$ These findings can be linked with our study since $\mathrm{Kondoh}^{28}$ revealed that Notch signaling suppressed MAPK/ERK Activity in myoblasts and inhibited the myogenic process. The Notch signaling pathway, which plays an important role in cell development and differentiation, is evolutionarily conserved in mammals. ${ }^{29}$ It also plays an important role in regulating both satellite cell homeostasis in quiescent muscles and myoblast development during muscle regeneration. ${ }^{30,31}$ Following activation of Notch, satellite cells can exit the cell cycle and return to quiescence, ${ }^{32}$ which may then help to maintain their stem cell function. In this study, we found that the Dll4-Notch-Csl - Hes1 axis was up-regulated, indicating that Notch signaling was activated after treatment with lidocaine (Figure S5). Notably, we found that the expression level of Pax7, a marker of muscle stem cells, was significantly increased after treatment with low concentrations $(0.01 \%$ or $0.02 \%)$ of lidocaine. Such events may promote a self-renewal and enlargement or maintenance of the satellite cell pool, which may have the effect of delaying future muscle repair. When using local anesthetics for treatment of strabismus, the tension of the EOMs sometimes increases after a longterm recovery period. ${ }^{24}$ We propose that after the initial damage, EOMs experience a delay in the repair of these muscles due to a maintenance or even enlargement of satellite cell pools. The subsequent repair may eventually form muscle fibers of increased strength to provide the necessary forces required for appropriate eye alignment. Based upon the results of this study, a new perspective on the drug therapy of strabismus is presented in which these drugs can be applied to promote the pluripotent capacity of muscle stem cells for the acute damage of EOMs. In this way, it would be possible to remodel EOMs to achieve an appropriate eye alignment, while avoiding potential side effects of surgery, such as conjunctival scars or limitations in ocular motility.

In conclusion, here we investigated some of the cellular/molecular mechanisms through which lidocaine affects myoblasts and the myotoxicity that results from local anesthetics. We found that lidocaine can arrest $\mathrm{C} 2 \mathrm{C} 12$ cells in G0/G1 phase of the cell cycle, inhibit myoblast migration and myogenic differentiation, processes which may be associated with activation and regulation of the Notch signaling pathway. To our knowledge, this represents the first study to systematically describe some of the mechanisms by which local anesthetics can affect muscle regeneration and the maintenance of satellite cells as demonstrated at cellular and molecular levels. Such an information is essential for future studies and drug therapy development in the treatment of strabismus.

\section{Statement of Ethics}

Informed consent, adhering to the tenets of the Declaration of Helsinki, was obtained from all participants or their guardians prior to the use of their samples in the study. This study was approved by the Institutional Review Board of the Zhongshan Ophthalmic Center. The Ethical Code is 2019KYPJ103. 


\section{Funding}

This work was supported by the National Natural Science Foundation of China (81670885). The funders had no role in study design, data collection and analysis, decision to publish, or preparation of the manuscript. No additional external funding was received for this study.

\section{Disclosure}

The authors report no financial conflict of interest and conflicts of interest in this work.

\section{References}

1. Piegeler T, Votta-Velis EG, Liu G, et al. Antimetastatic potential of amide-linked local anesthetics: inhibition of lung adenocarcinoma cell migration and inflammatory Src signaling independent of sodium channel blockade. Anesthesiology. 2012;117(3):548-559. doi:10.1097/ALN.0b013e3182661977

2. Li R, Xiao C, Liu H, Huang Y, Dilger JP, Lin J. Effects of local anesthetics on breast cancer cell viability and migration. BMC Cancer. 2018;18(1):666.

3. Chen J, von Bartheld CS. Role of exogenous and endogenous trophic factors in the regulation of extraocular muscle strength during development. Invest Ophthalmol Vis Sci. 2004;45(10):3538-3545.

4. Scott AB, Miller JM, Shieh KR. Treating strabismus by injecting the agonist muscle with bupivacaine and the antagonist with botulinum toxin. Trans Am Ophthalmol Soc. 2009;107:104-109.

5. Anderson BC, Christiansen SP, Grandt S, Grange RW, McLoon LK. Increased extraocular muscle strength with direct injection of insulin-like growth factor-I. Invest Ophthalmol Vis Sci. 2006;47 (6):2461-2467.

6. Öz Gergin Ö, Yıldız K, Bayram A, et al. Comparison of the myotoxic effects of levobupivacaine, bupivacaine, and ropivacaine: an electron microscopic study. Ultrastruct Pathol. 2015;39(3):169-176.

7. Oz Gergin O, Bayram A, Gergin IS, et al. Comparison of myotoxic effects of levobupivacaine, bupivacaine and ropivacaine: apoptotic activity and acute effect on pro-inflammatory cytokines. Biotech Histochem. 2019;94(4):252-260.

8. Zink W, Graf BM. Local anesthetic myotoxicity. Reg Anesth Pain Med. 2004;29(4):333-340.

9. Hussain N, McCartney CJL, Neal JM, Chippor J, Banfield L, Abdallah FW. Local anaesthetic-induced myotoxicity in regional anaesthesia: a systematic review and empirical analysis. $\mathrm{Br}$ J Anaesth. 2018;121(4):822-841.

10. Irwin W, Fontaine E, Agnolucci L, et al. Bupivacaine myotoxicity is mediated by mitochondria. J Biol Chem. 2002;277(14):12221-12227. doi:10.1074/jbc.M108938200

11. Hopker LM, Zaupa PF, Lima Filho AADS, et al. Bupivacaine and botulinum toxin to treat comitant strabismus. Arq Bras Oftalmol. 2012;75(2):111-115. doi:10.1590/S0004-27492012000200008

12. Hoyt CS. A problem! Now a solution? Br J Ophthalmol. 2007;91 (2):127-128.

13. Maurice JM, Gan Y, Ma FX, Chang YC, Hibner M, Huang Y. Bupivacaine causes cytotoxicity in mouse $\mathrm{C} 2 \mathrm{C} 12$ myoblast cells: involvement of ERK and Akt signaling pathways. Acta Pharmacol Sin. 2010;31(4):493-500.
14. Li R, Ma H, Zhang X, et al. Impaired autophagosome clearance contributes to local anesthetic bupivacaine-induced myotoxicity in mouse myoblasts. Anesthesiology. 2015;122(3):595-605.

15. Ohto H, Kamada S, Tago K, et al. Cooperation of six and eya in activation of their target genes through nuclear translocation of Eya. Mol Cell Biol. 1999;19(10):6815-6824.

16. Lazaro JB, Bailey PJ, Lassar AB. Cyclin D-cdk4 activity modulates the subnuclear localization and interaction of MEF2 with SRC-family coactivators during skeletal muscle differentiation. Genes Dev. 2002;16(14):1792-1805.

17. Lu J, McKinsey TA, Zhang CL, Olson EN. Regulation of skeletal myogenesis by association of the MEF2 transcription factor with class II histone deacetylases. Mol Cell. 2000;6(2):233-244.

18. Shen H, McElhinny AS, Cao Y, et al. The Notch coactivator, MAML1, functions as a novel coactivator for MEF2C-mediated transcription and is required for normal myogenesis. Genes Dev. 2006;20(6):675-688.

19. Ma X, Kuang X, Xia Q, et al. Covalent CDK7 Inhibitor THZ1 Inhibits Myogenic Differentiation. J Cancer. 2018;9(17):3149-3155.

20. Chen Z, Jin G, Lin S, et al. DNA methyltransferase inhibitor CDA-II inhibits myogenic differentiation. Biochem Biophys Res Commun. 2012;422(3):522-526.

21. Lin S, Shen H, Jin B, et al. Brief report: blockade of Notch signaling in muscle stem cells causes muscular dystrophic phenotype and impaired muscle regeneration. Stem Cells. 2013;31(4):823-828.

22. Bentzinger CF, Wang YX, Rudnicki MA. Building muscle: molecular regulation of myogenesis. Cold Spring Harb Perspect Biol. 2012;4 (2):a008342.

23. Reurink G, Goudswaard GJ, Moen MH, Weir A, Verhaar JA, Tol JL. Myotoxicity of injections for acute muscle injuries: a systematic review. Sports Med. 2014;44(7):943-956.

24. Bilgin B, Gursoy H, Basmak H, et al. The effects of bupivacaine injection and oral nitric oxide on extraocular muscle in the rabbit. Graefes Arch Clin Exp Ophthalmol. 2013;251(9):2227-2233.

25. Hofmann P, Metterlein T, Bollwein G, et al. The myotoxic effect of bupivacaine and ropivacaine on myotubes in primary mouse cell culture and an immortalized cell line. Anesth Analg. 2013;117 (3):634-640.

26. Goetsch KP, Myburgh KH, Niesler CU. In vitro myoblast motility models: investigating migration dynamics for the study of skeletal muscle repair. J Muscle Res Cell Motil. 2013;34(5-6):333-347.

27. Guyton DL. Strabismus complications from local anesthetics. Semin Ophthalmol. 2008;23(5):298-301.

28. Kondoh K, Sunadome K, Nishida E. Notch signaling suppresses p38 MAPK activity via induction of MKP-1 in myogenesis. J Biol Chem. 2007;282(5):3058-3065.

29. Li L, Tang P, Li S, et al. Notch signaling pathway networks in cancer metastasis: a new target for cancer therapy. Med Oncol. 2017;34 (10): 180 .

30. Liu L, Charville GW, Cheung TH, et al. Impaired Notch Signaling Leads to a Decrease in p53 Activity and Mitotic Catastrophe in Aged Muscle Stem Cells. Cell Stem Cell. 2018;23(4):544-556.e4.

31. Dumont NA, Wang YX, Rudnicki MA. Intrinsic and extrinsic mechanisms regulating satellite cell function. Development. 2015;142(9):1572-1581.

32. Kuang S, Kuroda K, Le Grand F, Rudnicki MA. Asymmetric self-renewal and commitment of satellite stem cells in muscle. Cell. 2007;129(5):999-1010. 


\section{Publish your work in this journal}

Drug Design, Development and Therapy is an international, peerreviewed open-access journal that spans the spectrum of drug design and development through to clinical applications. Clinical outcomes, patient safety, and programs for the development and effective, safe, and sustained use of medicines are a feature of the journal, which has also been accepted for indexing on PubMed Central. The manuscript management system is completely online and includes a very quick and fair peer-review system, which is all easy to use. Visit http://www. dovepress.com/testimonials.php to read real quotes from published authors. 